\title{
Betatron Radiation from Electron Beams in Plasma Focusing Channels
}

\author{
E. Esarey, P. Catravas, and W.P. Leemans \\ Center for Beam Physics, Ernest Orlando Lawrence Berkeley National Laboratory, University of \\ California, Berkeley CA 94720
}

\begin{abstract}
Spontaneous radiation emitted from an electron undergoing betatron motion is a plasma focusing channel is analyzed and applications to plasma wakefield accelerator experiments and to the ion channel laser (ICL) are discussed. Important similarities and differences between a free electron laser (FEL) and in an ICL are delineated. It is shown that the frequency of spontaneous radiation is a strong function of the betatron strength parameter $a_{\beta}$, which plays a similar role to that of the wiggler strength parameter in a conventional FEL. For $a_{\beta} \gtrsim 1$, radiation is emitted in numerous harmonics. Furthermore, $a_{\beta}$ is proportional to the amplitude of the betatron orbit, which varies for every electron in the beam. This places serious limits on the possibility of realizing an ICL.
\end{abstract}

\section{INTRODUCTION}

The propagation of electrons beams through plasmas is relevant to a variety of advanced accelerator [1] and novel radiation source applications [2]. Presently two experiments are underway at the Stanford Linear Accelerator Center (SLAC) that explore the interaction of an intense electron bunch with a plasma. These are the E-150 plasma lens experiment [3] and the E-157 plasma wakefield experiment [4]. In the E-157 experiments, a $30 \mathrm{GeV}$ electron beam of $2 \times 10^{10}$ electrons in a 0.65 $\mathrm{mm}$ long bunch is propagated through a $1.4 \mathrm{~m}$ long lithium plasma with an electron density up to $2 \times 10^{14} \mathrm{~cm}^{-3}$. The electron bunch propagates through the plasma in the so-called blowout regime [5], i.e., the initial beam density is greater than the plasma density. In this regime, the head of the bunch expels the plasma electrons and leaves behind a nearly uniform ion channel. The bunch length and plasma density are chosen such that the blown-out plasma electrons come crashing back to the axis near the tail of the bunch, thus driving a very large axial electric field, on the order of several $100 \mathrm{MV} / \mathrm{m}$, that can accelerate the electrons in the tail of the bunch.

One consequence of operating in the blowout regime is that the main body of the electron bunch resides in the nearly uniform ion channel, since the plasma 
electrons are blown out to approximately the plasma skin depth, $k_{p}^{-1}=c / \omega_{p}$, which is typically much greater than the bunch radius, where $\omega_{p}=\left(4 \pi n_{e} e^{2} / m_{e} c^{2}\right)^{1 / 2}$ is the plasma frequency and $n_{e}$ is the electron plasma density. Associated with the ion channel are very strong transverse fields, on the order of several thousand tesla per meter, that subsequently focus the body of the electron bunch. Since the initial beam radius $(50-100 \mu \mathrm{m})$ is much greater that the matched beam radius $(\sim 5 \mu \mathrm{m})$, the beam radius will undergo betatron oscillations as it propagates through the plasma [2], [3]. In the blowout regime, the radial space charge electric field is given [3] by $E_{r}=\left(e m_{e} / c^{2}\right) k_{p}^{2} r / 2$. At the edge of the beam, $r=r_{b}$, this can be written in practical units as

$$
E_{r}[\mathrm{MV} / \mathrm{m}]=9.06 \times 10^{-15} n_{e}\left[\mathrm{~cm}^{-3}\right] r_{b}[\mu \mathrm{m}]
$$

Likewise, in the blowout regime, the betatron wavelength is given by $\lambda_{\beta}=$ $(2 \gamma)^{1 / 2} \lambda_{p}$, where $\gamma$ is the relativistic factor of the electron and $\lambda_{p}=2 \pi / k_{p}$ is the plasma wavelength, which can be written as

$$
\lambda_{p}[\mathrm{~cm}]=3.34 \times 10^{6}\left(n_{e}\left[\mathrm{~cm}^{-3}\right]\right)^{-1 / 2} .
$$

Time integrated optical transition radiation has been used to study the transverse beam profile dynamics in the E-157 experiments [6]. Up to three betatron oscillations of the beam radius has been observed.

In addition to the blowout regime of the plasma wakefield accelerator, an accelerated electron bunch will experience transverse focusing forces in typical plasma-based accelerator configurations, such as the laser wakefield accelerator [1]. For example, in the laser wakefield accelerator that operates in the linear regime, the wakefield is often described by an electrostatic potential of the form $\Phi=\Phi_{0} \exp \left(-r^{2} / r_{p}^{2}\right) \cos k_{p}(z-c t)$, where $r_{p}$ is the radius of the wake and is proportional to radius of the drive beam. Notice that the axial electric field $E_{z}=-\partial \Phi / \partial z$ and the radial electric field $E_{r}=-\partial \Phi / \partial r$ are phased such that there exists a $\pi / 2$ region of axial phase $k_{p}(z-c t)$ that is both accelerating and focusing. An electron residing off-axis will undergo radial betatron oscillations about the axis due to the transverse focusing force of the wakefield. The magnitude of the focusing field near the axis is given by $\left|E_{r}\right|=2 r \Phi_{0} / r_{p}^{2}$, assuming $\cos k_{p}(z-c t)=1$, and the betatron wavelength is given by $\lambda_{\beta}=\pi r_{p}\left(2 \gamma / \hat{\Phi}_{0}\right)^{1 / 2}$, where $\hat{\Phi}_{0}=e \Phi_{0} / m_{e} c^{2}$ is the normalized amplitude of the wakefield. The density perturbation on axis associated with the wake is given by $\delta n_{e} / n_{e}=-\hat{\Phi}_{0}\left(1+4 / k_{p}^{2} r_{p}^{2}\right) \cos k_{p}(z-c t)$. Electron blowout near the axis occurs when $\hat{\Phi}_{0}=k_{p}^{2} r_{p}^{2} / 4$, assuming $k_{p} r_{p} / 2<1$.

As an electron undergoes betatron oscillations in a plasma focusing channel, it will emit synchrotron radiation [7]. In the limit of a small amplitude betatron oscillation, i.e., an electron displaced slightly from the axis, the wavelength of the synchrotron radiation is given by $\lambda=\lambda_{\beta} / 2 \gamma^{2}$, where $\lambda_{\beta}$ is the wavelength of the betatron oscillation and $\gamma$ is the relativistic factor of the electron. For plasma-based accelerators, this can easily be in the hard x-ray regime. For the E-157 experiment, $\lambda_{\beta} \sim 0.8 \mathrm{~m}$ and $\gamma=6 \times 10^{4}$, such that $\lambda \sim 0.1 \mathrm{~nm}$. 
The betatron motion in a focusing channel also forms the basis of the ion channel laser [2]. In the ion channel, radiation at the resonant wavelength $\lambda=\lambda_{\beta} / 2 \gamma^{2}$ can feed back on the electron beam, leading to axial bunching of the beam, and coherent amplification of the radiation. The amplification process is analogous to that in a free electron laser, with the betatron motion analogous to the wiggler motion in a free electron laser (FEL) [8]. It has been suggested that the ICL mechanism can further enhance the spontaneous synchrotron radiation in the E157 experiments [9], thus leading to partially coherent radiation near the $0.1 \mathrm{~nm}$ region. It is necessary that the details of the single particle synchrotron radiation in a plasma focusing channel be well understood, in order to access the prospects for the generation of self-amplified spontaneous emission (SASE) in an ICL.

In this article, spontaneous radiation emitted from an electron undergoing betatron motion in a plasma focusing channel is analyzed starting from basic principles. Application of these results to the E-157 experiment and to the ICL are examined. Important similarities and differences between SASE in an FEL and in an ICL are delineated. It is shown that the spontaneous radiation emitted along the axis of a plasma focusing channel from a single electron occurs near the resonant frequency

$\omega_{n}=2 \gamma_{z 0}^{2} n \omega_{\beta} /\left(1+a_{\beta} / 2\right)^{1 / 2}$, where $\gamma_{z 0}$ is the initial gamma factor for the electron entering the channel, $n$ is the harmonic number, $\omega_{\beta}=c k_{\beta}=2 \pi c / \lambda_{\beta}$ is the betatron frequency, $a_{\beta}=\gamma_{z 0} k_{\beta} r_{\beta}$ is the betatron strength parameter, and $r_{\beta}$ is the amplitude of the betatron orbit. The role of the betatron strength parameter $a_{\beta}$ is analogous to that of the wiggler strength parameter $a_{w}$ (or $K_{w}$ ) in FEL physics. In Ref. [2], the ICL was considered only in the limit $a_{\beta}^{2} \ll 1$. When $a_{\beta}^{2} \ll 1$, radiation is emitted primarily at the fundamental frequency $\omega=2 \gamma_{z 0}^{2} \omega_{\beta}$ and is independent of $a_{\beta}$. For $a_{\beta} \gtrsim 1$, however, radiation is emitted in numerous harmonics in which the resonant frequency is a strong function of $a_{\beta}$. This is the case in the E-157 experiments, in which $a_{\beta} \sim 2-50$. In an ideal FEL, the wiggler strength parameter $a_{w}$ is a constant (a function of only the magnetic field of the wiggler) for all of the beam electrons. However, in an ICL, $a_{\beta}=\gamma_{z 0} k_{\beta} r_{\beta}$ depends on both the electron energy $\gamma_{z 0}$ and the betatron amplitude $r_{\beta}$. Since $r_{\beta}$, and hence $a_{\beta}$, is different for every electron in a typical beam, this places serious limits on the possibility of realizing a SASE ICL.

\section{ELECTRON MOTION IN PLASMA FOCUSING CHANNELS}

The electron motion in a plasma focusing channel is governed by the relativistic Lorentz equation, which may be written in the form

$$
d \mathbf{u} / d c t=\nabla \hat{\Phi}
$$

where $\hat{\Phi}=e \Phi / m_{e} c^{2}$ is the normalized electrostatic potential of the focusing channel, $\mathbf{u}=\mathbf{p} / m_{e} c=\gamma \beta$ is the normalized electron momentum, and $\gamma=\left(1+u^{2}\right)^{1 / 2}=$ 
$\left(1-\beta^{2}\right)^{-1 / 2}$ is the relativistic factor. Here only the transverse focusing force of the plasma is considered. Near the axis, $r^{2} \ll r_{0}^{2}$, the space charge potential is assumed to have the form

$$
\hat{\Phi}=\hat{\Phi}_{0}\left(1-r^{2} / r_{0}^{2}\right),
$$

such that the normalized radial electric field is $\hat{E}_{r}=-\partial \hat{\Phi} / \partial r=2 \hat{\Phi}_{0} r / r_{0}^{2}$, where $\hat{\Phi}_{0}$ and $r_{0}$ are constants. The electrostatic potential is related to the electron plasma density by $\nabla^{2} \hat{\Phi}=k_{p}^{2}\left(n_{e} / n_{0}-1\right)$, where a uniform background of plasma ions of density $n_{0}$ is assumed. The maximum focusing field occurs in the when the plasma electrons are completely expelled (blown out) from the channel, $n_{e}=0$. Notice that in the blowout regime, $\hat{E}_{r}=k_{p}^{2} r / 2$, hence, $\hat{\Phi}_{0} / r_{0}^{2} \leq k_{p}^{2} / 4$.

Equation (4) implies the existence of two constants of the motion, $d u_{z} / d t=0$ and $d(\gamma-\hat{\Phi}) / d t=0$. Inside the focusing channel, the electron orbits (assuming the electron orbit lies in the $x-z$ plane) are given by

$$
\begin{gathered}
\tilde{\beta}_{x} \simeq k_{\beta} r_{\beta} \cos \left(k_{\beta} c t\right), \\
\tilde{x} \simeq r_{\beta} \sin \left(k_{\beta} c t\right), \\
\tilde{\beta}_{z} \simeq \beta_{z 0}\left(1-k_{\beta}^{2} r_{\beta}^{2} / 4\right)-\beta_{z 0}\left(k_{\beta}^{2} r_{\beta}^{2} / 4\right) \cos \left(2 k_{\beta} c t\right), \\
\tilde{z} \simeq z_{0}+\beta_{z 0}\left(1-k_{\beta}^{2} r_{\beta}^{2} / 4\right) c t-\beta_{z 0}\left(k_{\beta} r_{\beta}^{2} / 8\right) \sin \left(2 k_{\beta} c t\right),
\end{gathered}
$$

where

$$
k_{\beta}=\left(2 \hat{\Phi}_{0} / \gamma_{z 0} r_{0}^{2}\right)^{1 / 2}
$$

is the betatron wavenumber, $r_{\beta}$ is the constant amplitude of the betatron orbit, $u_{z}=u_{z 0}, \gamma_{z 0}=\left(1+u_{z 0}^{2}\right)^{1 / 2}, \beta_{z 0}=u_{z 0} / \gamma_{z 0}$ and $z_{0}$ is a constant. Equations (5)-(8) are the leading order contributions to the orbits, assuming $k_{\beta}^{2} r_{\beta}^{2} / 2 \ll 1$. Notice that in the blowout regime $\hat{\Phi}_{0}=k_{p}^{2} r_{0}^{2} / 4$ and the betatron wavenumber is given by $k_{\beta}=k_{p} /\left(2 \gamma_{z 0}\right)^{1 / 2}$.

For an ensemble of particles comprising an electron beam, the evolution of the RMS beam radius $r_{b}$ evolves via envelope equation [10]

$$
d^{2} r_{b} / d c t^{2}=\epsilon_{n}^{2} \gamma^{-2} r_{b}^{-3}-k_{\beta}^{2} r_{b},
$$

where $\epsilon_{n} \simeq \gamma r_{b} \theta_{b}$ is the normalized beam emittance, $\theta_{b}$ is the RMS beam angle, and $\gamma m_{e} c^{2}$ is the beam energy, where the effects of finite energy spread and space charge have been neglected. A matched beam occurs when $d^{2} r_{b} / d c t^{2}=0$, i.e., at a matched radius given by

$$
r_{b m}=\left(\epsilon_{n} / \gamma k_{\beta}\right)^{1 / 2},
$$

at which point the expansion of the beam due to finite emittance is balanced by the focusing forces of the plasma channel. For parameters typical of the E-157 experiment $\left(\epsilon_{n}=10 \mathrm{~mm}-\mathrm{mrad}, \gamma=6 \times 10^{4}\right.$, and $\left.\lambda_{\beta}=0.82 \mathrm{~m}\right), r_{b m}=4.7 \mu \mathrm{m}$. 


\section{SYNCHROTRON RADIATION}

The energy spectrum of the radiation emitted by a single electron in an arbitrary orbit $\tilde{\mathbf{r}}(t)$ and $\tilde{\beta}(t)$ can be calculated from the Lienard-Wiechert potentials [11],

$$
\frac{d^{2} I}{d \omega d \Omega}=\frac{e^{2} \omega^{2}}{4 \pi^{2} c}\left|\int_{-T / 2}^{T / 2} d t[\mathbf{n} \times(\mathbf{n} \times \tilde{\beta})] \exp [i \omega(t-\mathbf{n} \cdot \tilde{\mathbf{r}} / c)]\right|^{2}
$$

where $d^{2} I / d \omega d \Omega$ is the energy radiated per frequency, $\omega$, per solid angle, $\Omega$, during the interaction time, $T$, and $\mathbf{n}$ is a unit vector pointing in the direction of observation. It is convenient to introduce the spherical coordinates $(r, \theta, \phi)$ where $x=r \sin \theta \cos \phi, y=r \sin \theta \sin \phi, z=r \cos \theta$, and $\mathbf{n}$ is along the $\mathbf{e}_{r}$ direction. Using the betatron orbits given above, the radiation spectrum can be calculated with conventional techniques [12]. The resulting spectrum is given by

$$
\begin{aligned}
\frac{d^{2} I}{d \omega d \Omega}= & \sum_{n=1}^{\infty} \frac{e^{2} k^{2}}{4 \pi^{2} c}\left(\frac{\sin \bar{k} L / 2}{\bar{k}}\right)^{2} \\
& \cdot\left[C_{x}^{2}\left(1-\sin ^{2} \theta \cos ^{2} \phi\right)+C_{z}^{2} \sin ^{2} \theta-C_{x} C_{z} \sin 2 \theta \cos \phi\right],
\end{aligned}
$$

where

$$
\begin{aligned}
& C_{x}=k_{\beta} r_{\beta} \sum_{m=-\infty}^{\infty} J_{m}\left(\alpha_{z}\right)\left[J_{n+2 m-1}\left(\alpha_{x}\right)+J_{n+2 m+1}\left(\alpha_{x}\right)\right] \\
& C_{z}=\beta_{z 0} \sum_{m=-\infty}^{\infty} J_{m}\left(\alpha_{z}\right)\left\{2\left(1+k_{\beta}^{2} r_{\beta}^{2} / 4\right) J_{n+2 m}\left(\alpha_{x}\right)\right. \\
&\left.-\left(k_{\beta}^{2} r_{\beta}^{2} / 4\right)\left[J_{n+2 m-2}\left(\alpha_{x}\right)+J_{n+2 m+2}\left(\alpha_{x}\right)\right]\right\} \\
& \alpha_{z}= \frac{n\left(k / k_{n}\right)\left(a_{\beta}^{2} / 4\right) \cos \theta}{\left[\left(1+a_{\beta}^{2} / 2\right) \cos \theta+2 \gamma_{z 0}^{2}(1-\cos \theta)\right]} \\
& \alpha_{x}= \frac{n\left(k / k_{n}\right) 2 \gamma_{z 0} a_{\beta} \sin \theta \cos \phi}{\left[\left(1+a_{\beta}^{2} / 2\right) \cos \theta+2 \gamma_{z 0}^{2}(1-\cos \theta)\right]} \\
& \bar{k}=\alpha_{0} k-n k_{\beta} \\
& \alpha_{0}=1-\beta_{z 0}\left(1-k_{\beta}^{2} r_{\beta}^{2} / 4\right) \cos \theta
\end{aligned}
$$

and

$$
a_{\beta}=\gamma_{z 0} k_{\beta} r_{\beta}
$$

is the betatron strength parameter. Here, $L=c T$ is the interaction length, $k=$ $\omega / c$ is the radiation wavenumber, $n$ is the harmonic number, and $J_{m}$ are Bessel functions. For parameters typical of the E-157 experiment $\left(\gamma=6 \times 10^{4}\right.$ and $\lambda_{\beta}=$ $0.82 \mathrm{~m}), a_{\beta}=45$ for $r_{\beta}=r_{b}=100 \mu \mathrm{m}$ (typical unmatched beam radius) and $a_{\beta}=2.1$ for $r_{\beta}=r_{b m}=4.7 \mu \mathrm{m}$ (typical matched beam radius). 
Provided $N_{\beta} \gg 1$, radiation is emitted in a series of harmonics and is confined in a narrow bandwidth about the resonant frequency of each harmonic, where $N_{\beta}=L / \lambda_{\beta}$ is the number of betatron periods that the electron undergoes. The frequency width of the radiation spectrum for a given harmonic is determined by the resonance function $R_{n}(k)$, where

$$
R_{n}(k)=\left(\frac{\sin \bar{k} L / 2}{\bar{k} L / 2}\right)^{2}
$$

This function is sharply peaked about the resonant frequency, $\omega_{n}=c k_{n}$, given by $\bar{k}=0$,

$$
k_{n}=\frac{n k_{\beta}}{\alpha_{0}} \simeq \frac{2 \gamma_{z 0}^{2} n k_{\beta}}{\left[\left(1+a_{\beta}^{2} / 2\right) \cos \theta+2 \gamma_{z 0}^{2}(1-\cos \theta)\right]},
$$

where $\gamma_{z 0}^{2} \gg 1$ was assumed. The width of the spectrum, $\Delta \omega$, about $\omega_{n}$ is given by $\Delta \omega / \omega_{n}=1 / n N_{\beta}$. Furthermore, $R_{n}(k) \rightarrow \Delta \omega_{n} \delta\left(\omega-\omega_{n}\right)$ as $N_{\beta} \rightarrow \infty$. The angular width $\Delta \theta_{n}$ within which can be found radiation with frequencies in $\Delta \omega$ about $\omega_{n}$, for a single harmonic $n$, is given by $\Delta \theta_{n} \simeq\left(2 \Delta \omega / M_{0} \omega_{n}\right)^{1 / 2}$. Typically, for frequencies of interest, the synchrotron radiation is confined to a cone angle $\theta^{2} \ll 1$ and the resonant frequency can be approximated by

$$
\omega_{n} \simeq n M_{0} c k_{\beta} /\left(1+M_{0} \theta^{2} / 2\right),
$$

where $M_{0}=2 \gamma_{z 0}^{2} /\left(1+a_{\beta}^{2} / 2\right)$ is the relativistic Doppler upshift factor.

\section{On-Axis Radiation}

Of particular interest is the radiation emitted along the axis. Along the axis, $\theta=0$, only the odd harmonics are finite, i.e., the even harmonics vanish. Setting $\theta=0$ in the above expressions gives, for the $n^{t h}$ odd harmonic, $\alpha_{x}=0, \alpha_{z}=\alpha_{n}$, and

$$
\frac{d^{2} I_{n}(0)}{d \omega d \Omega}=2 e^{2} \frac{\omega}{\omega_{n}} k_{\beta} N_{\beta} M_{0}^{2} G_{n} F_{n}=\frac{4 e^{2}}{c} \frac{\omega}{\omega_{n}} \frac{\gamma_{z 0}^{2} N_{\beta}^{2} R_{n} F_{n}}{\left(1+a_{\beta}^{2} / 2\right)},
$$

where

$$
F_{n}\left(a_{\beta}\right)=n \alpha_{n}\left[J_{(n-1) / 2}\left(\alpha_{n}\right)-J_{(n+1) / 2}\left(\alpha_{n}\right)\right]^{2}
$$

is the harmonic amplitude function,

$$
\alpha_{n}=\frac{n\left(\omega / \omega_{n}\right) a_{\beta}^{2} / 4}{\left(1+a_{\beta}^{2} / 2\right)}
$$

and

$$
G_{n}(\omega)=\frac{R_{n}(k)}{\Delta \omega_{n}}=\frac{1}{\Delta \omega_{n}} \frac{\sin ^{2}\left[\pi n N_{\beta}\left(\omega / \omega_{n}-1\right)\right]}{\left[\pi n N_{\beta}\left(\omega / \omega_{n}-1\right)\right]^{2}}
$$


is the frequency spectrum function with the resonant frequency $\omega_{n}=n M_{0} c k_{\beta}$.

The energy radiated in the $n^{t h}$ backscattered harmonic depends on the function $F_{n}\left(a_{\beta}\right)$, Eq. $(25)$. For high harmonics, $n \gg 1, F_{n}$ becomes significant when $a_{\beta}^{2} \gg 1$. For $a_{\beta}^{2} \ll 1$, only the fundamental, $n=1$, is significant. Figure 1 shows a plot of $d^{2} I(0) / d \omega \delta \Omega$ versus $\omega / 2 \gamma_{z 0}^{2} \omega_{\beta}$ for the first four harmonics $(n=1,2$, 3 , and 4) with $N_{\beta}=4$. The solid curve shows the radiation from a single electron with $a_{\beta}=\gamma_{z 0} k_{\beta} r_{\beta}=4$, indicating that radiation is emitted in well-defined harmonics. The dashed (dotted) curve shows the spectrum integrated over a flattop (Gaussian) distribution of betatron amplitudes $r_{\beta}$ with a rms value satisfying $a_{\beta, r m s}=\gamma_{z 0} k_{\beta} r_{\beta, r m s}=4$. Figure 2 shows a blow-up of Fig. 1 . The effect of averaging over a distribution of electron orbits is clearly to smooth out the spectrum, since the frequency of the radiation emitted by a single electron is a strong function of $a_{\beta}$. Note that the fall-off on the right of the dashed (dotted) curve in Figs. 1 and 2 is artificial, since only the first four harmonic terms are included in this calculation of the spectrum.

An expression for the number of photons $\left(N_{n}\right)$ radiated per electron can be obtained for photons in a narrow bandwidth near the resonant frequency by dividing Eq. (24) by the energy per photon $\left(\hbar \omega_{n}\right)$. The total number of photons radiated per electron in the in the intrinsic bandwidth $\Delta \omega_{n}=\omega_{n} / n N_{\beta}$ about $\omega_{n}$ is given by integrating over this narrow frequency band and multiplying by the solid angle $2 \pi\left(\Delta \theta^{2} / 2\right)^{1 / 2}$, where $\Delta \theta \simeq\left(2 \Delta \omega_{n} / M_{0} \omega_{n}\right)^{1 / 2}$, which gives

$$
N_{n} \simeq 4 \pi \alpha_{f}\left(\Delta \omega_{n} / \omega_{n}\right)\left(N_{\beta} / n\right) F_{n}\left(a_{\beta}\right)
$$

where $\alpha_{f}$ is the fine structure constant.

\section{Ultra-Intense Behavior}

For values of $a_{\beta}^{2} \ll 1$, the scattered radiation will be narrowly peaked about the fundamental resonant frequency, $\omega_{1}$, given by Eq. (22) with $n=1$. As $a_{\beta}$ approaches unity, scattered radiation will appear at harmonics of the resonant frequency as well, $\omega_{n}=n \omega_{1}$. When $a_{\beta} \gg 1$, high harmonic $(n \gg 1)$ radiation is generated and the resulting synchrotron radiation spectrum consists of many closely spaced harmonics. Finite variations in the parameter $a_{\beta}=\gamma_{z 0} k_{\beta} r_{\beta}$ within an electron beam can broaden the linewidth and cause the spectrum to overlap. Hence, in the ultra-intense limit, i.e., $a_{\beta} \gg 1$, the gross spectrum appears broadband, and a continuum of radiation is generated which extends out to a critical frequency, $\omega_{c}$, beyond which the radiation intensity diminishes. The critical frequency can be written as $\omega_{c}=n_{c} M_{0} \omega_{\beta}$, where $n_{c}$ is the critical harmonic number. It is possible to calculate $n_{c}$ by examining the radiation spectrum, Eqs. (13)-(15), in the ultraintense limit, $a_{\beta} \gg 1$.

Asymptotic properties of the radiation spectrum for large harmonic numbers, $n \gg 1$, can be analyzed using conventional methods [12]. In particular, the asymp- 


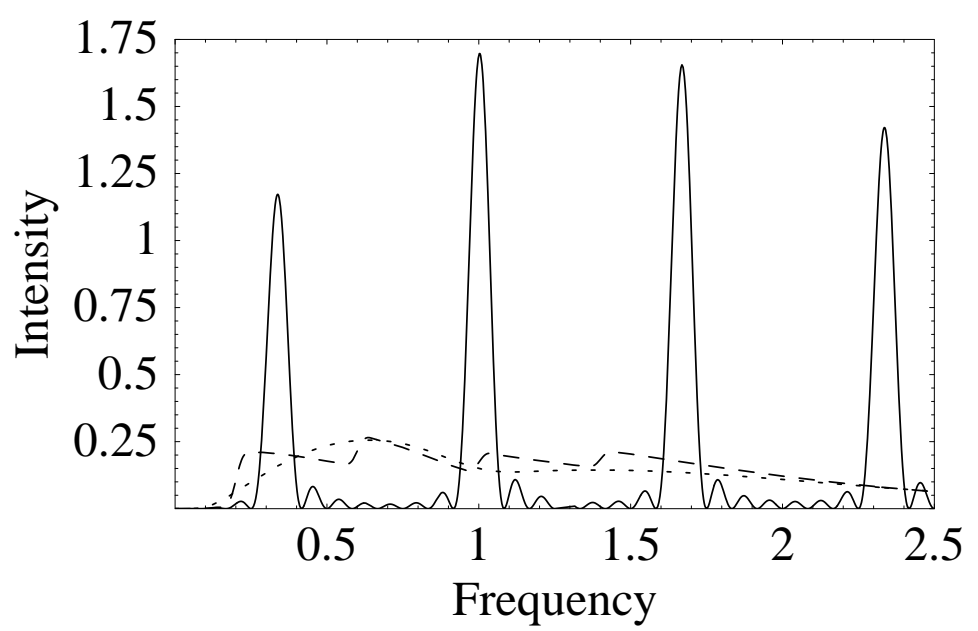

FIGURE 1. Normalized spectrum $d^{2} I(0) / d \omega \delta \Omega$ (arbitrary units) versus $\omega / 2 \gamma_{z 0}^{2} \omega_{\beta}$ for the first four harmonics $(n=1,2,3$, and 4$)$ with $N_{\beta}=4$. The solid curve shows the radiation from a single electron with $a_{\beta}=\gamma_{z 0} k_{\beta} r_{\beta}=4$. The dashed (dotted) curve shows the spectrum integrated over a flat-top (Gaussian) distribution of betatron amplitudes $r_{\beta}$ with $a_{\beta, r m s}=\gamma_{z 0} k_{\beta} r_{\beta, r m s}=4$.

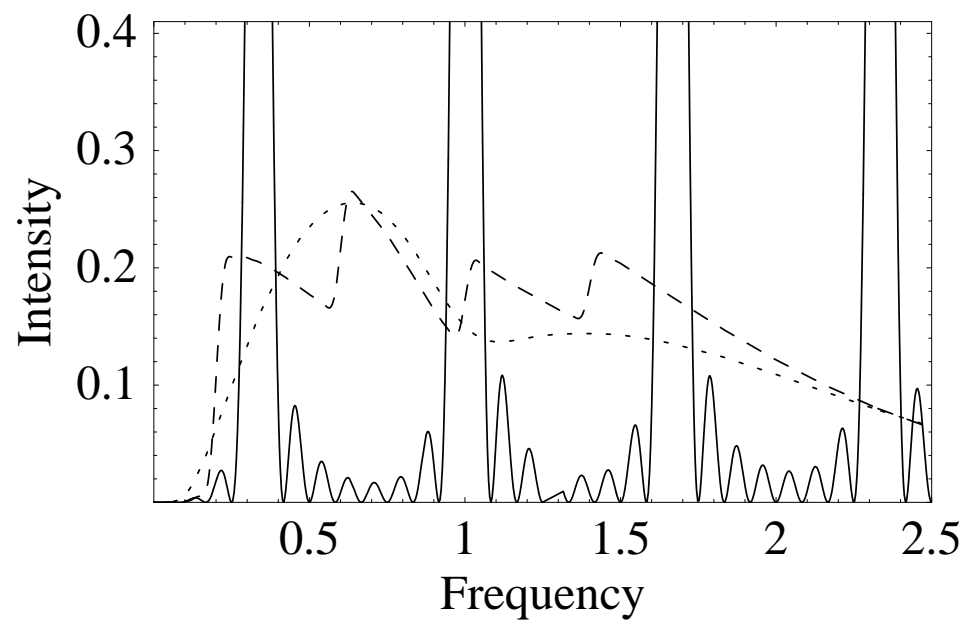

FIGURE 2. Blow-up of Fig. 1.

totic spectrum in the vertical direction $(\phi=\pi / 2)$ is given by

$$
\frac{d^{2} I}{d \omega d \Omega} \simeq N_{\beta} \frac{6 e^{2}}{\pi^{2} c} \frac{\gamma_{z 0}^{2} \zeta^{2}}{\left(1+\gamma_{z 0}^{2} \theta^{2}\right)}\left[\frac{\gamma_{z 0}^{2} \theta^{2}}{\left(1+\gamma_{z 0}^{2} \theta^{2}\right)} K_{1 / 3}^{2}(\zeta)+K_{2 / 3}^{2}(\zeta)\right]
$$

where

$$
\begin{gathered}
\zeta=\frac{\omega}{\omega_{c}}\left(1+\gamma_{z 0}^{2} \theta^{2}\right)^{3 / 2}, \\
\omega_{c}=n_{c} M_{0} \omega_{\beta} \simeq 3 a_{\beta} \gamma_{z 0}^{2} \omega_{\beta}
\end{gathered}
$$




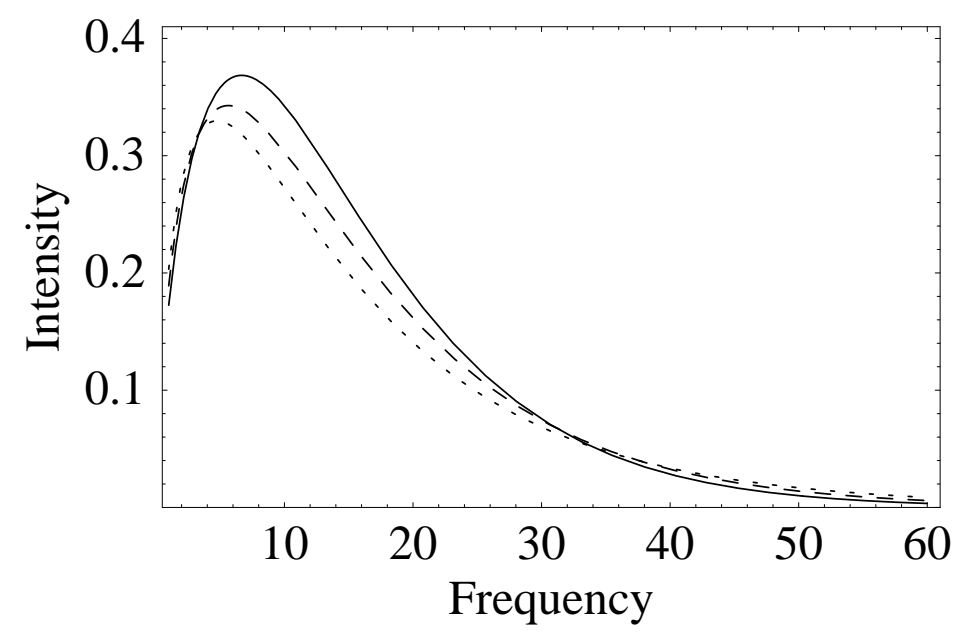

FIGURE 3. The function $Y(\xi)=\xi^{2} K_{2 / 3}^{2}(\xi)$ versus $\omega / 2 \gamma_{z 0}^{2} \omega_{\beta}$. The solid curve shows the radiation from a single electron with $a_{\beta}=\gamma_{z 0} k_{\beta} r_{\beta}=10$. The dashed (dotted) curve shows the spectrum integrated over a flat-top (Gaussian) distribution of betatron amplitudes $r_{\beta}$ with $a_{\beta, r m s}=\gamma_{z 0} k_{\beta} r_{\beta, r m s}=10$.

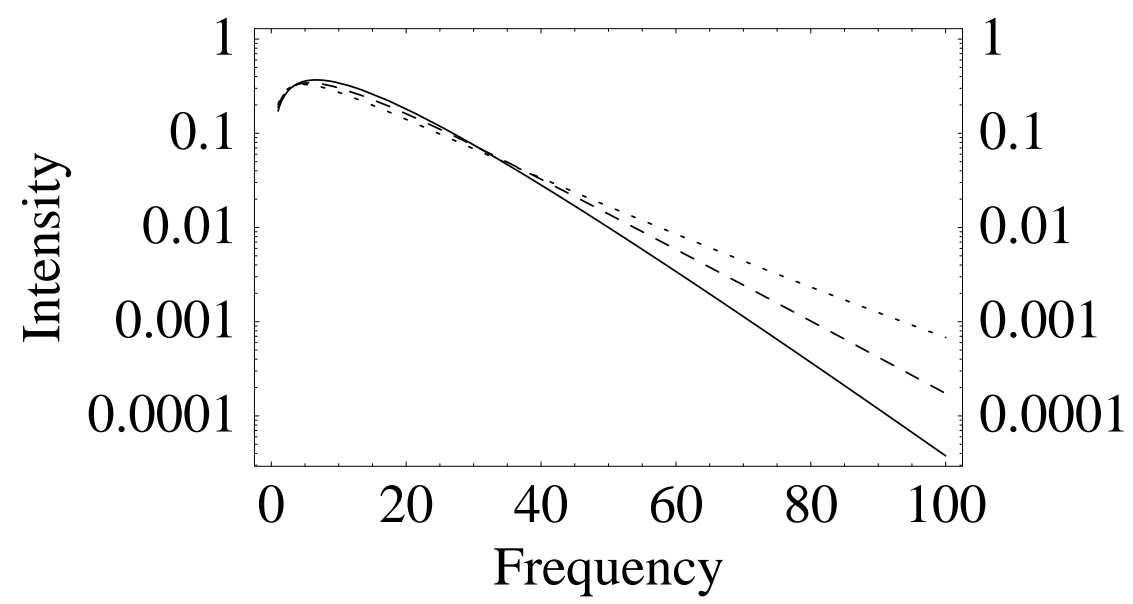

FIGURE 4. Figure 3 plotted on a log scale.

is the critical frequency,

$$
n_{c} \simeq 3 a_{\beta}^{3} / 4
$$

is the critical harmonic number, and $M_{0} \simeq 4 \gamma_{z 0}^{2} / a_{\beta}^{2}$. For E-157-like parameters $\left(\gamma=6 \times 10^{4}, \lambda_{\beta}=0.82 \mathrm{~m}\right.$, and $\left.a_{\beta}=45\right), n_{c} \simeq 6.8 \times 10^{4}$ and $\lambda_{c}=2 \pi c / \omega_{c} \simeq$ $1.7 \times 10^{-12} \mathrm{~m}$.

Along the axis $\theta=0, d^{2} I(0) / d \omega d \Omega \sim \xi^{2} K_{2 / 3}^{2}(\xi)$, where $\xi=\omega / \omega_{c}$. The function $Y(\xi)=\xi^{2} K_{2 / 3}^{2}(\xi)$ is maximum at $\xi=1 / 2$ and decreases rapidly for $\xi>1$. The peak intensity occurs along the axis $\theta=0$, at approximately the critical frequency, 
$\omega \simeq \omega_{c}$, i.e., $n \simeq n_{c}=3 a_{\beta}^{3} / 4$. Half the total power is radiated at frequencies $\omega<\omega_{c} / 2$ and half at $\omega>\omega_{c} / 2$. For harmonics below $n_{c}\left(\omega \ll \omega_{c}\right)$, the radiation intensity increases as $\left(\omega / \omega_{0}\right)^{2 / 3}$, and above $n_{c}\left(\omega \gg \omega_{c}\right)$, the radiation intensity decreases exponentially as $\exp \left(-2 \omega / \omega_{c}\right)$. Furthermore, for $\omega \ll \omega_{c}$, the scattered radiation at a fixed frequency is confined to an angular spread $\Delta \theta=\left(\omega_{c} / \omega\right)^{1 / 3} / \gamma_{z 0}$ about $\theta=0$, whereas for $\omega>\omega_{c}, \Delta \theta=\left(\omega_{c} / 3 \omega\right)^{1 / 2} / \gamma_{z 0}$. The average angular spread for the frequency integrated spectrum in the vertical direction $(\phi=\pi / 2)$ is is $\theta_{v}=\left\langle\theta^{2}\right\rangle^{1 / 2} \sim 1 / \gamma_{z 0}$. In the horizontal direction $(\phi=0)$, emission is confined to the angle $\theta_{h} \sim a_{\beta} / \gamma_{z 0}$.

A plot of the function $Y(\xi)=\xi^{2} K_{2 / 3}^{2}(\xi)$ versus $\omega / 2 \gamma_{z 0}^{2} \omega_{\beta}$ is shown in Fig. 3 (linear scale) and Fig. 4 (log scale). The solid curve shows the radiation from a single electron with $a_{\beta}=\gamma_{z 0} k_{\beta} r_{\beta}=10$. The dashed (dotted) curve shows the spectrum integrated over a flat-top (Gaussian) distribution of betatron amplitudes $r_{\beta}$ with a rms value satisfying $a_{\beta, r m s}=\gamma_{z 0} k_{\beta} r_{\beta, r m s}=10$. To calculate these averages, the quantity $\xi=\omega / \omega_{c}$ has been approximated by $\xi \simeq\left(\omega / 2 \gamma_{z 0}^{2} \omega_{\beta}\right)\left(1+3 a_{\beta} / 2\right)^{-1}$, since the asymptotic form for the spectrum is not accurate when $a_{\beta}<1$, i.e., Figs. 3 and 4 are inaccurate in the region $\omega / 2 \gamma_{z 0}^{2} \omega_{\beta} \lesssim 1$.

\section{RADIATED POWER AND ELECTRON ENERGY LOSS}

The power radiated by a single electron, $P_{s}$, undergoing relativistic quiver motion in an intense laser field can be calculated from the relativistic Larmor formula [11]

$$
P_{s}=\left(2 e^{2} / 3 c\right) \gamma^{2}\left[(d \mathbf{u} / d t)^{2}-(d \gamma / d t)^{2}\right] .
$$

Using the orbits described above, the power radiated by a single electron undergoing betatron motion is given by

$$
\left\langle P_{s}\right\rangle \simeq r_{e} m_{e} c^{3} \gamma_{z 0}^{2} k_{\beta}^{2} a_{\beta}^{2} / 3
$$

where an averaging was performed over the betatron period, $\gamma_{z 0}^{2} \gg 1$ was assumed, and $r_{e}=e^{2} / m_{e} c^{2}$ is the classical electron radius.

The rate at which a single electron loses energy due to radiating is given by $W_{\text {loss }}^{\prime}=\left\langle P_{s}\right\rangle / c$, i.e.,

$$
W_{\text {loss }}^{\prime} \simeq r_{e} m_{e} c^{2} \gamma_{z 0}^{2} k_{\beta}^{2} a_{\beta}^{2} / 3 .
$$

In the blowout regime, $k_{\beta} \sim n_{0}^{1 / 2} \gamma_{0 z}^{-1 / 2}$, and the rate of energy loss scales as $W_{\text {loss }}^{\prime} \sim$ $n_{0}^{2} \gamma_{z 0}^{2} r_{\beta}^{2}$. In addition, if the betatron amplitude is equal to the matched beam radius $r_{b}=\left(\epsilon_{n} / \gamma_{z 0} k_{\beta}\right)^{1 / 2}$, the energy loss scales as $W_{\text {loss }}^{\prime} \sim \epsilon_{n} \gamma_{z 0}^{3} k_{\beta}^{3} \sim \epsilon_{n} n_{0}^{3 / 2} \gamma_{z 0}^{3 / 2}$. For example, in the blowout regime at a density $n_{0}=2 \times 10^{14} \mathrm{~cm}^{-3}\left(\lambda_{p}=0.24\right.$ $\mathrm{cm}$ ) and a beam energy of $\gamma_{z 0}=6 \times 10^{4}$, an electron with a betatron amplitude of $r_{\beta}=100 \mu \mathrm{m}\left(a_{\beta}=45\right)$ would lose energy at a rate of $W_{\text {loss }}^{\prime}=0.2 \mathrm{MeV} / \mathrm{m}$. 


\section{ION CHANNEL LASER}

Under special conditions, e.g., sufficiently high electron beam quality, selfamplified spontaneous emission (SASE) can occur whereby the incoherent synchrotron radiation emitted by the electrons is amplified via the ion channel laser (ICL) mechanism [2]. In the ICL instability, the radiation beats with the betatron motion to create an axial $\mathbf{v} \times \mathbf{B}$ (i.e., ponderomotive) force that leads to bunching of the electron beam and growth of the radiation field. This can lead to large levels of semi-coherent or coherent radiation. In SASE, the incoherent, spontaneous radiation acts as a seed for the instability, in a manner analogous to the SASE mode of operation in a free electron laser (FEL) [8].

There are important differences between the ICL and FEL mechanisms, however, that limit the SASE mode of operation. For electrons undergoing betatron motion in a plasma focusing channel, the resonant frequency of the radiation emitted along the axis is given by $\omega=2 \gamma_{z 0}^{2} n \omega_{\beta} /\left(1+a_{\beta}^{2} / 2\right)$, as indicated by Eq. (23). For an FEL, the resonant frequency is $\omega=2 \gamma_{z 0}^{2} n \omega_{w} /\left(1+a_{w}^{2} / 2\right)$, where $\omega_{w}=c k_{w}=2 \pi c / \lambda_{w}$, $\lambda_{w}$ is the wiggler wavelength, $a_{w}=e B_{w} / k_{w} m_{e} c^{2}$ is the wiggler strength, and $B_{w}$ is the field amplitude of the wiggler magnet. In an ideal FEL, $a_{w}$ is a constant since all the electrons experience the same value of $B_{w}$. This is contrast to the focusing channel, in which $a_{\beta}=\gamma_{z 0} k_{\beta} r_{\beta}$ is a function of both the electron energy $\gamma_{z 0}$ and the radial position of the electrons via the betatron amplitude $r_{\beta}$. If a monoenergetic beam of finite radius is injected into a focusing channel (without any special tapering), electrons at different radii will have different betatron amplitudes $r_{\beta}$, different values of $a_{\beta}$, and hence different resonant frequencies.

Furthermore, for an ideal FEL with a planar wiggler of the form $\mathbf{B}=$ $B_{w} \cos \left(k_{w} z\right) \mathbf{e}_{x}$, all of the the beam electrons wiggle in the same plane with the same amplitude, i.e., $\mathbf{u}_{\perp}=a_{w} \cos \left(k_{w} z\right) \mathbf{e}_{x}$. Consequently, radiation emitted by all the electrons will have similar polarization. This is contrast to the focusing channel, in which the betatron motion, and hence the synchrotron radiation, will have a variety of polarizations in the $x-y$ plane, depending on the position and angle of the electron as it enters the channel. Hence, to amplify radiation of a given frequency and polarization in a focusing channel, only those beam electrons with the proper values of $\gamma_{z 0}$ and $r_{\beta}$ with be resonant with the radiation, and only a subset of these will have the proper polarization. This is contrast to an ideal FEL, in which all the electrons in a mono-energetic beam are resonant with the radiation field with the proper polarization.

It is straightforward to quantify some the conditions necessary for SASE to occur in a plasma focusing channel. In the following discussion, it is assumed that $k_{\beta}^{2} r_{\beta}^{2} \ll$ 1. Consider an ideal mono-energetic electron beam of radius $r_{b}$ injected into a focusing channel such that the beam centroid is along the $z$ axis. A electron moving along the axis would have a betatron amplitude of $r_{\beta}=0$, whereas an electron residing at the edge of the beam would have a betatron amplitude of

$r_{\beta}=r_{b}$. For the beam to emit radiation along the axis with a narrow bandwidth $\Delta \omega / \omega \ll 1$, it is necessary that $a_{\beta}^{2} \ll 1$ for all the electrons. This implies that the 
radiation wavelength satisfy $\lambda>\pi r_{b} / \gamma$. For a matched beam with a normalized emittance $\epsilon_{n}$, the matched-beam radius is given by $r_{b m}=\left(\epsilon_{n} / \gamma k_{\beta}\right)^{1 / 2}$, and the condition $a_{\beta}^{2} \ll 1$ implies

$$
\lambda \gg \pi \epsilon_{n} / \gamma
$$

It is interesting to note the similarity of this condition with that usually required of a SASE FEL [8], $\lambda>4 \pi \epsilon_{n} / \gamma$.

The condition $\Delta \omega / \omega \ll 1$, however, is not sufficient for the SASE process to occur. A more stringent condition is that the normalized axial energy spread $\Delta \gamma_{z} / \gamma_{z}$ be small compared to the so-called Pierce or gain parameter $\rho$, i.e., $\Delta \gamma_{z} / \gamma_{z} \ll \rho$, where by analogy with an FEL,

$$
\rho=\left[\frac{a_{\beta} k_{p b}}{4 \gamma^{3 / 2} k_{\beta}} F_{\Delta}^{2}\left(a_{\beta}\right)\right]^{2 / 3},
$$

where $k_{p b}=4 \pi n_{b} e^{2} / m_{e} c^{2}, n_{b}$ is the beam density, and

$$
F_{\Delta}\left(a_{\beta}\right)=J_{0}\left(\frac{a_{\beta}^{2} / 4}{1+a_{\beta}^{2} / 2}\right)-J_{1}\left(\frac{a_{\beta}^{2} / 4}{1+a_{\beta}^{2} / 2}\right) .
$$

In terms of the beam current $I_{b}=e c \pi n_{b} r_{b}^{2}$, and evaluating the expression for $\rho$ at $r_{\beta}=r_{b}$, gives

$$
\rho=\left(I_{b} F_{\Delta}^{4} / 4 \gamma I_{A}\right)^{1 / 3},
$$

where $I_{A}=m_{e} c^{3} / e=17 \mathrm{kA}$. Using the equations of motion for an electron in a focusing channel, Eqs. (5)-(8), the normalized energy spread is given by $\Delta \gamma_{z} / \gamma_{z} \simeq$ $a_{\beta}^{2} / 4$, for a beam with a centroid along the axis. Hence, $\Delta \gamma_{z} / \gamma_{z}<\rho$ implies $a_{\beta}^{2}<4 \rho$ or $\lambda>\pi r_{\beta} /\left(2 \gamma \rho^{1 / 2}\right)$. For a matched beam, this gives

$$
\lambda>\frac{\pi \epsilon_{n}}{4 \gamma \rho} .
$$

This is considerably more stringent that the usual FEL constraint $\lambda>4 \pi \epsilon_{n} / \gamma$, since typically $\rho \ll 1$. For the parameters of the E-157 experiment, $\rho \simeq 5 \times 10^{-3}$.

In principle, it may be possible to tailor the energy distribution and radial profile of the beam such that a greater fraction of the beam electrons are in resonance with the radiation field. For example, consider a mono-energetic, very narrow beam of width $\Delta r_{b}$ injected off-axis such that the centroid of the beam executes betatron oscillations of amplitude $r_{\beta}=r_{b 0}$ with $r_{b 0} \gg \Delta r_{b}$ [13]. In this case, all of the electrons in the beam would undergo approximately the same betatron orbit and would have approximately the same value for $a_{\beta}$, i.e., the spread in $a_{\beta}$ is given by $\Delta a_{\beta} / a_{\beta} \simeq \Delta r_{b} / r_{b 0}$. In this case the condition $\Delta \omega / \omega \ll 1$ implies $\Delta r_{b} / r_{b 0} \ll\left(1+a_{\beta}^{2} / 2\right) / a_{\beta}^{2}$, which in principle, could be easily satisfied. The more stringent condition, $\Delta \gamma_{z} / \gamma_{z}<\rho$, implies $\Delta r_{b} / r_{b 0}<2 \rho / a_{\beta}^{2}$, which could be satisfied for sufficiently small values of $a_{\beta}$. 
Even if the condition $\Delta \gamma_{z} / \gamma_{z}<\rho$ is satisfied, it is not clear that the SASE process would occur. In a conventional FEL, SASE requires that a number of conditions be satisfied (in addition to $\Delta \gamma_{z} / \gamma_{z}<\rho$ ) [8], i.e., $\epsilon_{n}<\gamma \lambda / 4 \pi, N_{\beta} \lambda_{\beta} \gg L_{G}, L_{G}<L_{R}$, and $N_{\beta} \lambda<L_{e}$, where $N_{\beta}$ is the number of betatron oscillations, $L_{g} \simeq 0.046 \lambda_{\beta} / \rho$ is the gain length, $L_{R}=\pi w_{0}^{2} / \lambda$ is the Rayleigh length of the radiation with spot size $w_{0}$, and $L_{e}$ is the electron bunch length. Furthermore, for the case of an ICL driven by a narrow beam with a centroid undergoing betatron oscillations, it is likely that the gain (i.e., $\rho$ ) is reduced since the geometric overlap between the electron beam and the radiation is reduced, due to the betatron motion of the centroid. Such novel ICL configurations require a detailed analysis.

\section{SUMMARY}

Spontaneous radiation emitted from an electron undergoing betatron motion is a plasma focusing channel was analyzed starting from basic principles. Application of these results to the E-157 experiment and to the ICL were examined. Important similarities and differences between SASE in an FEL and in an ICL were delineated. In particular, the spontaneous radiation emitted along the axis of a plasma focusing channel from a single electron occurs near the resonant frequency

$\omega_{n}=2 \gamma_{z 0}^{2} n \omega_{\beta} /\left(1+a_{\beta}^{2} / 2\right)^{1 / 2}$. The role of the betatron strength parameter $a_{\beta}$ is analogous to that of the wiggler strength parameter $a_{w}$ (or $K_{w}$ ) in FEL physics. In Ref. [2], the ICL was considered only in the limit $a_{\beta}^{2} \ll 1$. When $a_{\beta}^{2} \ll 1$, radiation is emitted primarily at the fundamental frequency $\omega=2 \gamma_{z 0}^{2} \omega_{\beta}$ and is independent of $a_{\beta}$. For $a_{\beta} \gtrsim 1$, however, the resonant frequency is a strong function of $a_{\beta}$ and radiation is emitted in numerous harmonics extending out to the critical harmonic number $n_{c}=3 a_{\beta}^{3} / 4$. This is the case in the E-157 experiments, in which $a_{\beta} \sim 2-50$.

In an ideal FEL, the wiggler strength parameter $a_{w}$ is a constant (a function of only the magnetic field of the wiggler) for all of the beam electrons. However, in an ICL, $a_{\beta}=\gamma_{z 0} k_{\beta} r_{\beta}$ depends on both the electron energy $\gamma_{z 0}$ and the betatron amplitude $r_{\beta}$. Since $r_{\beta}$, and hence $a_{\beta}$, is different for every electron in a typical beam, this places serious limits on the possibility of realizing a SASE ICL. For an electron beam center about the axis with a radius $r_{b}$ and $a_{\beta}\left(r_{b}\right)>1$, the radiation from the beam is no longer emitted at discrete harmonics as it would be from a single electron with $r_{\beta}=r_{b}$. Rather, since $0<a_{\beta} \lesssim a_{\beta}\left(r_{b}\right)$ for the electrons in the beam, the resulting radiation is in the form of a broad continuum as indicated by Figs. 1 and 2 , even for the case of an initially mono-energetic beam. In the limit $a_{\beta}^{2} \ll 1$, the radiation from the beam could be nearly monochromatic at the fundamental frequency. The condition $a_{\beta}^{2} \ll 1$ implies $\lambda \gg \pi \epsilon_{n} / \gamma$ for a matched beam, which is similar to the criterion $\lambda>4 \pi \epsilon_{n} / \gamma$ often quoted for a SASE FEL. The condition $a_{\beta}^{2} \ll 1$, however, is not sufficient to insure that the SASE ICL process will occur. A more stringent condition for the occurrence of SASE is on the axial energy spread of the beam within the focusing channel, i.e., $\Delta \gamma_{z} / \gamma_{z} \ll \rho$, where $\rho$ is the effective Pierce (or gain) parameter. Again, since $r_{\beta}$ varies across the beam, there 
exists a large energy spread $\Delta \gamma_{z} / \gamma_{z} \simeq a_{\beta}^{2} / 4$. The condition $\Delta \gamma_{z} / \gamma_{z} \ll \rho$ implies $\lambda>\pi \epsilon_{n} /(4 \gamma \rho)$ for a matched beam. Since typically $\rho \ll 1$, this restriction on the radiated wavelength $\lambda>\pi \epsilon_{n} /(4 \gamma \rho)$ is much more stringent than that in a conventional SASE FEL. Furthermore, the betatron orbits in a typical beam in a focusing channel are not polarized in the same plane as they are in a conventional FEL. This also can reduce the gain in a SASE ICL. These arguments, however, assumed an untailored electron beam centered about the channel axis. It may be possible to relax this constraint on the radiated wavelength in a SASE ICL by appropriately tailoring the electron beam, for example, a narrow electron beam injected off-axis such that all of the beam electron execute approximately the same betatron orbit. Such novel ICL configurations require further analysis to access their viability.

\section{ACKNOWLEDGMENTS}

The authors acknowledge useful conversations with the participants of the working group on plasma wakefield accelerators and with the members of the E-157 collaboration. This work was supported by the Department of Energy under contract No. DE-AC-03-76SF0098.

\section{REFERENCES}

1. For a review see, E. Esarey et al., IEEE Trans. Plasma Sci. 24, 252 (1996).

2. D.H. Whittum, A.M. Sessler, and J.M. Dawson, Phys. Rev. Lett. 64, 2511 (1990); D.H. Whittum, Phys. Fluids B 4, 730 (1992).

3. J. Ng et al., SLAC Preprint, SLAC-PUB-8501 (2000); SLAC E-150 web site, URL http://www.slac.stanford.edu/exp/e150.

4. M.J. Hogan et al., Phys. Plasmas 7, 2241(2000); SLAC E-157 web site, URL http://www.slac.stanford.edu/grp/arb/e157.

5. J.B. Rosenzweig et al., Phys. Rev. A 44, 6189 (1991).

6. P. Catravas et al., Proc. 1999 Particle Accelerator Conf., Ed. by A. Luccio and W. Mackay (IEEE, Piscataway NJ, 1999), pp. 2111-2113.

7. W.A. Barletta et al., Nuc. Instr. Meth. A 423, 256 (1999).

8. Handbook of Accelerator Physics and Engineering, Edited by A.W. Chao and M. Tigner (World Scientific, Singapore, 1999).

9. S. Wang and C. Joshi, private communication.

10. M. Reiser, Theory and Design of Charged Particle Beams (Wiley, New York, 1994).

11. J.D. Jackson, Classical Electrodynamics, 2nd ed. (Wiley, New York, 1975), Chap. 14.

12. E. Esarey, S.K. Ride, and P. Sprangle, Phys. Rev. E 48, 3003 (1993).

13. In collaboration with the working group on plasma wakefield accelerators, this workshop. 